$$
\text { Corf }-9307116--1
$$

LA-UR- $\quad 93-2486$
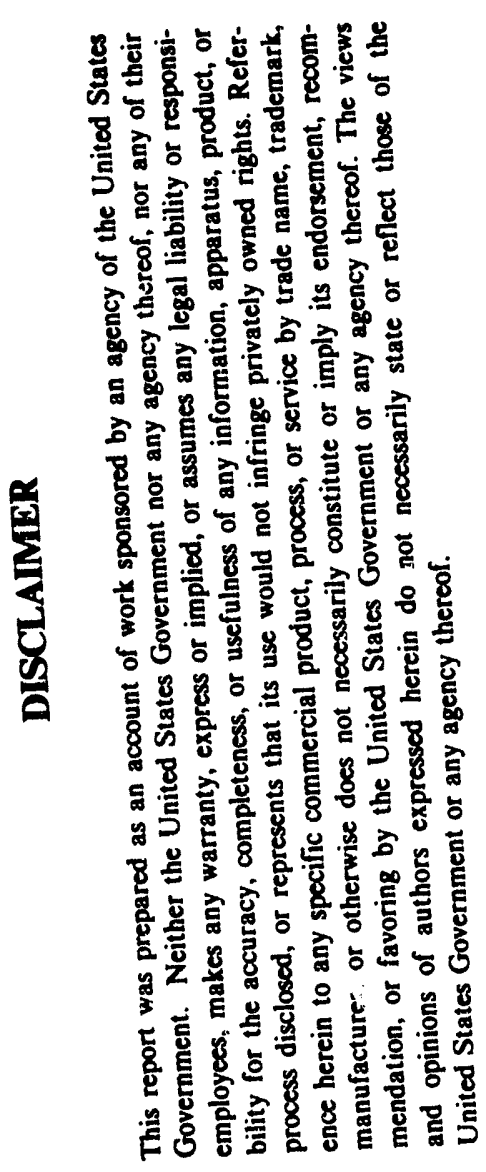

Title:

ELECTRON IMPACT POLARIZATION AND CORRELATION PROPERTIES OF THE INERT GASES

AUG $05 \quad 1 ., 3$

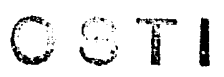

Submitted to:

D. C. Cartwright, G. Csanak, L. E. Machado, and

G. D. Meneses

Polarization and Correlation in Electronic \& Atomic Collisions

Bielefeld, Germany

July 29-31, 1993

( published in 8 für Physik D)
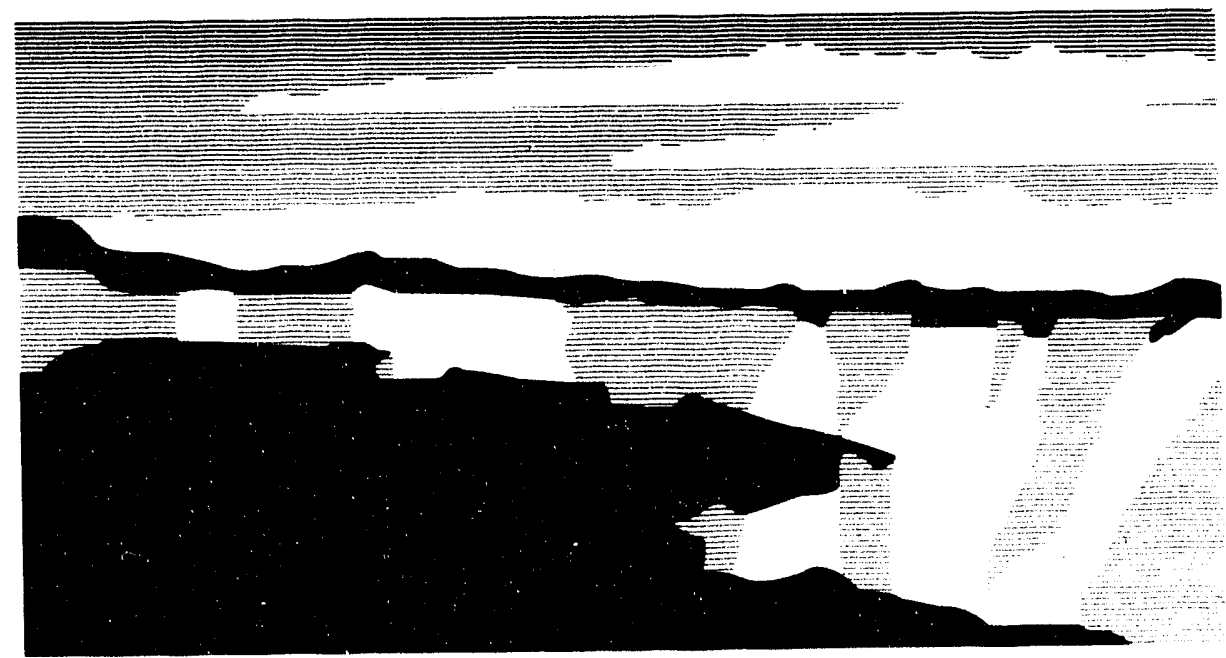

Los Alamos National Laboratory, an aftirmative action/equal opportunity employer, is operated by the University of California for the U.S. Department of Energy Los Alamos National Laboratory, an aftirmatice the Government retains a nonexclusive, royalty-free license to under contract W-7405-ENG-36. By acceptance of this article, the publisher recognizes that U.S. Government purposes. The Los Alamos Natignal Laboratory publish or reproduce the published form of this contribution, or to allow others to do so, for U.S. Government purposes. The
requests that the publisher identify this article as work performed under the auspices of the U.S. Department of Energy. 


\section{ELECTRON IMPACT POLARIZATION AND CORRELATION PROPERTIES OF THE INERT GASES}

G. Csanak and D. C. Cartwright Los Alamos National Laboratory Los Alamos, NM 87545

\section{E. Machado} Federal University of São Carlos São Carlos, Brazil

\section{G. D. Meneses}

University of Campinas

Campinas, Brazil 


\begin{abstract}
For the heavier rare-gas targets, $\mathrm{Ne}, \mathrm{Ar}, \mathrm{Kr}$, there is now a reasonable amount of experimental electron impact coherence parameter data available for excitation of the lowest $J=1$ states. Theoretical results for those rare-gas targets, have been restricted to distorted-wave approximation (DWA) type theories.

We present a systemization of the experimental data and compare them with available theoretical results. In the case of the heavy rare gases, we compare the experimental and theoretical data available for the three species, $\mathrm{Ne}, \mathrm{Ar}, \mathrm{Kr}$, in order to identify trends. We compare the experimental data with results from available theories (mainly DWA type) and discuss the importance of spin-orbit coupling effects and "shell" effects. We present our point-of-view as to the physical picture that is emerging from all collisional data, and conclude by recommending future experimental and theoretical activities that will, from our perspective, provide new insight into the physics of these processes.
\end{abstract}




\section{INTRODUCTION}

Electron-impact excitation studies on heavy rare-gas targets remain of interest even today. This is partially due to the great practical importance of these studies. Electron impact excitation of $\mathrm{Ar}$ and $\mathrm{Kr}$ atoms e.g. plays an important role in the modeling of the KrF laser-system (see articles in Cartwright [1]). The heavy rare-gas targets also provide an excellent testing ground for the study on the role of various physical effects in the electron collision process. In the case of neon target, for example, the spin-orbit coupling effect for the continuum electron can be neglected (Schackert [2], Walker [3]) while the spin-orbit coupling effect can be substantial in some of the excited states of neon (see Condon and Shortley [4]) such as the ${ }^{1} P_{1}$ and ${ }^{3} P_{1}$ states. This makes the heavy-rare gas targets excellent systems for the study of the role of target spin-orbit coupling in electron-impact excitation processes. It is important from the basic collisionphysics point of view that for these targets not only electron impact integral cross sections (ICSs) and differential cross sections (DCSs), but also electron impact coherence parameters (EICPs) have been reported from electron-photon coincidence measurements (reviewed recently by Becker, Crowe, and McConkey [5]). Furthermore, the measurement of spin-asymmetry-functions (Hanne [6]) is scheduled in the near future (Gay [7]). This means that for the heavy rare-gas targets, very detailed electron-collision physics information is available or will soon be available, which will serve as a stringent test on collisional theories. The purpose of the present work is to supplement the information that was obtained previously on electron impact excitation of heavy rare gases from first-order many-body theory (FOMBT) calculations (Padial et al. [8], Machado et al. [9, 10], da Paixão et al. [11, 12], Meneses et al. [13, 14]). The plan of this paper is as follows: Sec. II describes the theoretical model and gives the formulas to be used and Sec. III discusses the results obtained. 


\section{Theoretical Model}

The theoretical model used in this work is the same as the one used in our earlier works on electron impact excitation of argon (Padial et al. [8]), of neon (Machado et al. $[9,10]$ and of krypton (Meneses et al. $[13,14])$. The scattering model used is that of firstorder many-body theory (FOMBT) originally introduced for electron-atom inelastic scattering by Csanak et al. [15] and discussed in detail for its application for heavy raregas targets by Padial et al. [8]. The FOMBT has been shown to be one of many possible forms of a distorted wave approximation (DWA) by Rescigno et al. [16]. A special feature of the FOMBT as a DWA is that the distorted waves for both the incident and scattered electrons are calculated in the static-exchange field of the ground state of the target. Spin-orbit coupling effects in the continuum electron wave functions were neglected in our calculations. The representation of the target states in most cases is the same as in the earlier works quoted above. In this scheme the ${ }^{1} \mathrm{~S}_{0}$ ground state and the $n s^{\prime}[1 / 2]_{0}^{0}\left({ }^{3} \mathrm{P}_{0}\right)$ and $n s[3 / 2]_{2}^{0}\left({ }^{3} \mathrm{P}_{2}\right)$ excited states are described in the LSJ coupling formalism, whereas the $n s^{\prime}[1 / 2]_{1}^{0}\left("{ }^{\prime 1} P_{1} "\right)$ and $n s[3 / 2]_{1}^{0}\left({ }^{3} P_{1} "\right)$ states are represented as linear combinations of $L S J$ coupled ${ }^{1} \mathrm{P}_{1}$ and ${ }^{3} \mathrm{P}_{1}$ states in the form

$$
\begin{aligned}
& \left|n s^{\prime}[1 / 2]_{1}^{0} ; M_{J}\right\rangle=b\left|{ }^{1} P_{1} ; M_{J}\right\rangle+a\left|{ }^{3} P_{1} ; M_{J}^{\prime}\right\rangle \\
& \left.\left.\left|n s[3 / 2]_{1}^{0} ; M_{J}\right\rangle=-a||^{1} P_{1} ; M_{J}\right\rangle+b \beta^{3} P_{1} ; M_{J}\right\rangle
\end{aligned}
$$

with $a=0.266$ and $b=0.964$ for neon $(n=3)$, with $a=0.450$ and $b=0.893$ for Argon $(n=4)$, and with $a=0.730$ and $b=-0.683$ for Krypton $(n=5)$, obtained from Cowan [17]. Here we use the intermediate coupling notation of Moore [18] and put the closest LSJ coupled term in parentheses. (The same notation was used in our earlier works.) In other cases we used the CATS, ACE, and TAPS codes developeri at Los Alamos National Laboratory (Abdallah et al. [19], Clark et al. [20-23]) that allowed a more accurate 
representation of the wave-functions. The calculational procedure for the T-matrices for the various transitions is the same as in the earlier works of Padial et al. [8], Machado et al. $[9,10]$, and Clark et al $[22,23]$, thus it will not be given here.

In the present work we shall report electron impact coherence parameters for the excitation of the $3 s^{\prime}[1 / 2]_{1}^{0}$ and $3 s^{2}[3 / 2]_{1}^{0}$ states of neon, and $5 s^{\prime}[1 / 2]_{1}^{0}$ and $5 s[3 / 2]_{1}^{0}$ states of krypton using the FOMBT along with the representation of these wave-functions given by Eqs. (1a, b). In addition, we shall report spin-orbit coupling studies for neon and argon targets, and - dependence and Z-dependence studies via the use of the CATS, ACE, and TAPS codes. The relevant expressions for these physical quantities will be discussed now.

Following Bartschat [24], we shall define the electron scattering amplitude for the excitation of the $J_{1}, M_{1}$ excited state of the target by an incident electron of momentum $\overrightarrow{k_{1}}$ and spin $m_{0}$ and scattered electron of momentum $\overrightarrow{k_{1}}$ and spin $m_{1}$ as

$$
f\left(M_{1} m_{1} ; m_{0}\right)=\left\langle J_{1} M_{1} ; \overrightarrow{k_{1}} m_{1}|T| S_{0} ; \overrightarrow{k_{0}} m_{0}\right\rangle
$$

where $\left|{ }^{1} S_{0} ; \overrightarrow{k_{0}} m_{0}\right\rangle$ and $\left|J_{1} M_{1} ; \overrightarrow{k_{1}} m_{1}\right\rangle$ refer to the state vectors of the electron plus atom system before and after the collision, respectively, and $\mathrm{T}$ is the electron scattering operator. If we denote by $\rho_{m^{\prime}{ }^{\prime} m_{0}}$ the spin density matrix of the incident electron beam (for unpolarized electrons, $\rho_{m_{0}^{\prime} m_{0}}=1 / 2 \delta_{m_{0}^{\prime} m_{0}}$ ) then the density matrix of the combined electron plus atom system after the collision can be given by the formula,

$$
\rho_{m_{1}^{\prime} m_{1}}^{M_{1}^{\prime} M_{1}}=\sum_{m^{\prime} m_{0}} f\left(M_{1}^{\prime} m_{1}^{\prime} ; m_{0}^{\prime}\right) f^{*}\left(M_{1} m_{1} ; m_{0}\right) \rho_{m^{\prime} m_{0}}
$$


If the incident electron beam is unpolarized and if the spins of the scattered electrons are not detected, then we can define the reduced atomic density matrix in the form,

$$
\rho_{M_{1} M_{1}}^{a t}=\left\langle f\left(M_{1}\right) f^{*}\left(M_{1}\right)\right\rangle=\sum_{m_{1}} \rho_{m_{1} m_{1}}^{M_{1} M_{1}}=\frac{1}{2} \underset{m_{0} m_{1}}{\sum} f\left(M_{1} m_{1} ; m_{0}\right) f^{*}\left(M_{1} m_{1} ; m_{0}\right)
$$

The electron impact coherence parameters (EICP's) can now be defined conveniently in terms of the reduced atomic density matrix. One set of such parameters $\lambda, \Delta, \bar{\chi}, \varepsilon$ was introduced by da Paixão et al. [11] via the equations,

$$
\begin{aligned}
& \lambda=\frac{\sigma(0)}{\sigma(0)+2 \sigma(1)} \\
& \cos \Delta=\frac{\left|\left\langle f(1) f^{*}(0)\right\rangle\right|}{\sqrt{\sigma(0) \sigma(1)}} \\
& \bar{\chi}=\arg \left\{\left\langle f(1) f^{*}(0)\right\rangle\right\} \\
& \cos \varepsilon=-\frac{\left\langle f(1) f^{*}(-1)\right\rangle}{\sigma(1)}
\end{aligned}
$$

where

$$
\sigma(M)=\left\langle f(M) f^{*}(M)\right\rangle
$$

is the electron impact excitation cross section for magnetic sub-level $\mathrm{M}$, and

$$
\sigma=\sum_{M} \sigma(M)
$$


is the magnetic-sublevel summed differential cross section.

Another set of parameters that can be used in place of the above parameters are the Stokes parameters: $P_{1}, P_{2}, P_{3}$, and $P_{4}$. For a detailed discussion of these latter parameters see e.g. Andersen, Gallagher, Hertel [25] and Becker, Crowe and McConkey [5]. The $P_{1}, P_{2}, P_{3}$, Stokes parameters can be determined by an electron-photon coincidence experiment where the photon-detector is placed perpendicular to the collision plane, while $\mathrm{P}_{4}$ can be measured in a similar experiment with the photon-detector being in the collision plane. The modulus $|P|$ of the total polarization vector $P=\left(\mathrm{P}_{1}, \mathrm{P}_{2}, \mathrm{P}_{3}\right)$ gives the degree of polarization of the emitted radiation.

These Stokes parameters can be expressed in terms of the $\lambda, \bar{\chi}, \varepsilon, \Delta$ parameters by the formulas, (Andersen, Gallagher, Hertel [25], Khakoo and McConkey [26]),

$$
\begin{gathered}
P_{1}=\frac{\lambda(3+\cos \varepsilon)-(1+\cos \varepsilon)}{(1+\cos \varepsilon)+\lambda(1-\cos \varepsilon)} \\
P_{2}=\frac{-4[\lambda(1-\lambda)]^{1 / 2} \cos \Delta \cos \bar{\chi}}{(1+\cos \varepsilon)+\lambda(1-\cos \varepsilon)} \\
P_{3}=\frac{4[\lambda(1-\lambda)]^{1 / 2} \cos \Delta \sin \bar{\chi}}{(1+\cos \varepsilon)+\lambda(1-\cos \varepsilon)} \\
P_{4}=\frac{(\cos \varepsilon-1)+\lambda(3-\cos \varepsilon)}{(1-\cos \varepsilon)+\lambda(1-\cos \varepsilon)}
\end{gathered}
$$


Still another set of parameters, denoted by $\left(\gamma, L_{\perp}^{+}, P_{L}, \rho_{o o}\right)$ was introduced by Hermann and Hertel [27]. These latter parameters can be expressed in terms of parameters already introduced by the formulas,

$$
\begin{aligned}
& \gamma=\frac{1}{2} \arg \left(P_{1}+i P_{2}\right) \\
& L_{\perp}^{+}=-P_{3} \\
& P_{L}=\left(P_{1}^{2}+P_{2}^{2}\right)^{1 / 2} \\
& \rho_{o o}=\frac{1}{2}(1-\lambda)(1-\cos \varepsilon)
\end{aligned} .
$$

$\rho_{o o}$ can also be expressed in terms of the $P_{1}$ and $P_{4}$ parameters in the form,

$$
\rho_{\infty o}=\frac{\left(1+P_{1}\right)\left(1-P_{4}\right)}{4-\left(1-P_{1}\right)\left(1-P_{4}\right)}
$$




\section{Results and Discussion}

\section{A. Neon}

First we shall discuss our FOMBT results for the neon $3 s^{\prime}[1 / 2]_{1}^{0}$ and $3 s[3 / 2]_{1}^{0}$, EICPs. Fig. 1 shows our recent results for the DCSs for the excitation of the $3 s[3 / 2]_{1}^{0}, 2$ and $3 s^{\prime}[1 / 2]_{0}^{0}, 1$ levels in neon, along with the experimental data of Register et al. [28]. This figure clearly shows the good agreement of the present calculation with the experimental data, especially for the excitation of the $J=1$ levels. In Fig. 2 we compare our present FOMBT results for the $P_{1}, P_{2}$, and $P_{3}$ Stokes parameters and for the $P_{L}$ and $\gamma$ parameters in the case of the $\mathrm{J}=1$ levels excitation with the experimental data of Khakoo and McConkey [26].

This figure shows that FOMBT gives excellent agreement with experiment for the $P_{2}$ parameter practically for all angles measured and for the $\mathrm{P}_{1}, \mathrm{P}_{3}$ and $\gamma$ parameters for small scattering angles. The sign of the $P_{3}$ parameter agrees with that of the experiment for all scattering angles where comparison is possible. Fig. 3 shows the present results for the $\mathrm{P}_{3}$ and $\mathrm{P}_{4}$ parameters, for the excitation of both $\mathrm{J}=1$ levels in neon for incident electron energies $E=20,30,50$, and $80 \mathrm{eV}$. It can be seen immediately that sometimes these parameters change drastically as a function of energy, especially from $E=20 \mathrm{eV}$ to $\mathrm{E}=30 \mathrm{eV}$.

Further discussion on the comparison of our results to other theoretical results, and experimental data, can be found in Zheng and Becker [35].

\section{B. Krypton}

Our present results for the DCS for the excitation of the 5s, 5s' levels of krypton at $\mathrm{E}=50 \mathrm{eV}$ incident electron energy are shown in Fig. 4 where they are compared with the experimental data of Trajmar, et al. [30]. It can be seen again that excellent agreement is obtained with the experiment, especially for the excitation of the $J=1$ levels. The lowest panel of Fig. 12 shows our FOMBT results for the $P_{1}$ and $P_{2}$ parameters for incident electron energy $E=50 \mathrm{eV}$ in the case of the excitation of the $5 s^{\prime}[1 / 2]_{1}^{C}$ level of krypton 
where our present FOMBT results are compared with the distorted wave approximation (DWA) results of Bartschat and Madison [31] and with first-Born approximation (FBA) results as well as with the experimental data of Zheng and Becker [32]. From these figures it can be seen that for the quantities plotted at these energies, there is excellent agreement between the FOMBT results and experiment.

A more detailed discussion on the comparison of our results to other theories, and to the experimental data, can be found in Zheng and Becker [32].

\section{Comparative Studies}

In this section we report results from calculations using the CATS, ACE, and TAPS codes for some comparative studies.

\section{Spin-orbit coupling effects}

Fig. 5 shows the importance of spin-orbit-coupling effect in the excited state wave-function for the DCS in the case of the excitation of the " $3 \mathrm{P}_{1}$ " and " $1 \mathrm{P}_{1}$ " levels of neon. It can be seen immediately that the spin-orbit coupling has a drastic effect on the DCS for the excitation of the " $3 \mathrm{P}_{1}$ " level of neon even though the mixing coefficient for the ${ }^{1} \mathrm{P}_{1}$ coefficient (see upper panel) is quite small. This is, of course, due to the fact that the ${ }^{1} \mathrm{P}_{1}$ component brings in the possibility of a direct excitation of the " $3 \mathrm{P}_{1}$ " level.

Fig. 6 shows the importance of the spin-orbit coupling effect in the $P_{3}$ Stokes parameter for the electron impact excitation of the " $3 \mathrm{P}_{1}$ " level of neon and argon. It can be immediately seen that the spin-orbit coupling effect has a very large effect on the $P_{3}$ parameter for " $3 \mathrm{P}_{1}$ " excitation, in fact the spin-orbit coupled results for $\mathrm{P}_{3}$ are practically identical for "3 $\mathrm{P}_{1}$ " and " $\mathrm{P}_{1}$ " excitations (especially for argon and for $\vartheta<40^{\circ}$ scattering angles) showing a decisive influence of the ${ }^{1} P_{1}$ component in the wave-function. 


\section{Principal quantum number dependence}

Fig. 7 shows the principal quantum number $(n)$ dependence of the $P_{1}, P_{2}$, and $P_{3}$ Stokes parameters in the case of the excitation of $n s^{\prime}[1 / 2]_{1}^{0}, n=3,4,5$, and 6 levels of neon by $\mathrm{E}=80 \mathrm{eV}$ energy incident electron. It can be seen immediately that the Stokes parameters are practically independent from the principal quantum number as was found to be the case for helium $n^{1} P$ and $n^{3} P$ excitations. (See Csanak and Cartwright $[33,34]$.)

If $\mathrm{n}$-independence of the EICP's is indeed true, then the measurement of the EICP's can be affected by detecting radiation from higher lying $n s^{\prime}[1 / 2]_{1}^{0}$ or $n s^{\prime}[3 / 2]_{1}^{0}$ levels to lower lying excited levels when infrared or visible photon detectors could be used which would be especially advantageous for the measurement of the $\mathrm{P}_{3}$ parameter.

\section{Atomic number dependence}

Fig. 8 shows the DCS for the excitation of the $2^{1 P}$ level of He and $n s^{\prime}[1 / 2]_{1}^{0}$, $(\mathrm{n}=3,4,5)$ levels of $\mathrm{Ne}, \mathrm{Ar}$, and $\mathrm{Kr}$. We can immediately see, the increasingly pronounced diffraction (or interference) pattern with the increase of the atomic numbers due to the increasing number of partial waves with larger phase shifts that contribute to the DCS.

Fig. 9 shows identical studies for the $\gamma$ and $L_{\perp}$ parameters and Fig. 10 for the $\mathrm{P}_{3}$ parameter. It can be seen here also that with the increase of the atomic number the interference pattern becomes more complicated. It is important to point out that these latter quantities are ratios of two separate entities and interference effects appear in both the numerator and denominator, resulting in an even more complicated interference-effect in the ratio. Fig. 11 shows our results from identical studies for the $\rho_{o o}$ parameter. It can be immediately seen that the magnitude of this parameter, which characterizes the importance of angular momentum transfers to the target via a spin-flip process, increases with the increase of atomic numbers. This is obviously due to the increasing importance of the spin-orbit coupling effect in the final target state. 


\section{Conclusions}

Our results show that FOMBT gives from good to excellent results for the EICP's in the case of the excitation of the $\mathrm{J}=1$ levels of rare gases and that spin-orbit coupling effects in the target states are very important, even for neon. We found, as was the case for He, that the EICP's are practically independent of the principal quantum number and, for the heavier rare gases (argon, krypton), they are very similar for the "1 $P_{1}$ " and " ${ }^{2} P_{1}$ " levels. These two theoretical predictions should be tested experimentally. The good FOMBT results for both DCS's and EICP's indicate that many-body perturbation theory (MBPT) is a rapidly convergent approach for the calculation of these quantities and that the next higher order MBPT (such as second order MBPT) might give experimentally accurate results.

The excellent detailed, and authoritative, review of Becker, Crowe, and MicConkey [3] contains further conclusions and suggestions on this subject. However, experimental EICP data at larger scattering angles are needed (for several energies) and second order MBPT calculations need to be performed.

\section{Acknowledgments}

The authors want to acknowledge the financial support of the U.S. DOE, the NSF (OIP) and the CNPa (Brazil). They are grateful to Drs. R. D. Cowan, J. B. Mann, J. Abdallah, Jr., and R.E.H. Clark for participation in the development of the Los Alamos atomic physics and collisional codes and sharing their new computational algorithms. The authors particularly want to thank Bill McConkey, Kurt Becker, Al Stauffer and Sandor Trajmar for sharing the details associated with their results in advance of their publication, and for numerous stimulating discussions. 


\section{References}

1. Cartwright, D. C., Iriertial Confinement Fusion at Los Alamos, Volume II. LA-UR-89-2675, (1989)

2. Schackert, K. Z., Phys. 213, 316 (1968)

3. Walker, D. W., Adv. Phys. 20, 257 (1971)

4. Condon, E. V., and Shortley, G. H., The Theory of Atomic Spectra.Cambridge: Cambridge University Press, 1935

5. Becker, K., Crowe, A., McConkey, J. W., J. Phys. B 25.3885 (1992)

6. Hanne, F. G., Phys. Rep. 25,95 (1983)

7. Gay, T. J. Private communication to the authors (1989)

8. Padial, N. T., Meneses, G. D., da Paixão, F. J., Csanak, Gy., Cartwright, D. C., Phys. Rev. A23, 2194 (1984)

9. Machado, L. E., Leal, E. P., Csanak, Gy., J. Phys. B 20, 5541 (1987)

10. Machado, L. E., Leal, E. P., Csanak, G., Phys. Rev. A29, 1811 (1984)

11. da Paixão, F. J., Padial, N. T., Csanak, Gy, Blum, K., Phys Rev. Letts. 45, 1164 (1980)

12. da Paixão, F. J., Padial, N. T., Csanak, G., Phys Rev. A30 1697 (1984)

13. Meneses, G. D., da Paixão, F. J., Padial, N. T., Phys. Rev. A32, 156 (1985)

14 Meneses, G. D., da Paixão, F. J., Padial, N. T., Plys. Rev. A34, 675 (1986)

15. Csanak, Gy., Taylor, H. S., Yaris, R., Phys. Rev. A3. 1322 (1971)

16. Rescigno, T. N., McCurdy, C. W., and McKoy, V., J. Phys. B 1, 2396 (1974)

17. Cowan, R. D., Private communications to the authors, (1980)

18. Moore, C. E., Atomic Energy Levels Washington, D.C.: U.S. Government Printing Office (1948)

19. Abdallah, J., Clark, R. E. H., Cowan, R. D., Los Alamos Manual No. LA-11436-MI (unpublished) (1988)

20. Clark, R.E.H., Abdallah, J., Csanak, G., Mann, J. B., and Cowan, R. D., Los Alamos Manual No. LA-11436-MII (unpublished) (1988)

21. Clark, R.E.H., Abdallah, J. Kramer, S.P., Los Alamos Manual No. LA-11436-MIII (unpublished) (1988) 
22. Clark, R.E.H., Abdallah, J., Csanak, G., Kramer, S. P., Phys. Rev. A40, 2935 (1989)

23. Clark, R.E.H., Csanak, G., Abdallah, J., Phys. Rev. A44, 2874 (1991)

24. Bartschat, K., Phys. Rep. 180, 1 (1989)

25 Andersen, N., Gallagher, J. W., Hertel, I. V., Phys. Rep. 1651 (1988)

26. Khakoo, M. McConkey, J. W., J. Phys. B. 20, 5541 (1987)

27. Hermann, H. W., Hertel, I.V., Comments: At. Mol. Phys. 12, 61 (1982), ibid 12, 127 (1982)

28. Register, D. F., Trajmar, S., Steffensen, G., Cartwright, D. C., Phys. Rev. A29, 1793 (1984)

29. Corr, J. J., van der Burgt, P.J.M., Plessis, P., Khakoo, M. A., Hammond, P., McConkey, J. W., J. Phys. B24, 1069 (1991)

30. Trajmar, S., Srivastava, S. K., Tanaka, H., Nishimura, H., Phys. Rev. A23, 2167 (1981)

31. Bartschat, K., Madison, D. H., J. Phys. B20. 5839 (1987)

32. Zheng, S.R. and Becker, K., J. Phys. B26, 517 (1993)

33. Csanak, G. and Cartwright, D.C. Phys. Rev. A34, 93 (1986)

34. Csanak, G. and Cartwright, D. C. in Electronic and Atomic Collisions (H. B. Gilbody, W. R. Newell, I. H. Read, A. C. Smight, eds., Elseizer Science Publishers, B. V. 1988) p. 177

35. Zheng, S. R, and Becher, K. Z. Physik D23, 137 (1982)

36. Danjo, A., Koike, T., Kani T. Sugahara, H., Takahashi, A., and Nishimara, H., J. Phys. B18, L595 (1985)

37. McAdams, R., Hollywood, M.T., Crowe, A., and Williams, J. F., J. Phys B13, 3691 (1980) 


\section{Figure Captions}

Fig. 1 Differential cross sections for electron impact excitation of neon for $\mathrm{E}=50 \mathrm{eV}$ incident electron energy.

Solid line: Present results.

Shaded regions: Experimental results (with error bars) of Register et al [28].

Fig. 2 Stokes parameters $\mathrm{P}_{1}, \mathrm{P}_{2}, \mathrm{P}_{3}$, and $\mathrm{P}_{\mathrm{L}}$ and $\gamma$ parameters for electron impact excitation of neon.

Solid lines with filled circles: FOMBT results for neon ${ }^{1} P_{1}$ excitation at $E=80 \mathrm{eV}$ incident electron energy.

Dashed lines with empty circles: FOMBT results for neon ${ }^{3} \mathrm{P}_{1}$ excitation at $80 \mathrm{eV}$ incident electron energy.

Shaded regions: Experimental results of Khakoo and McConkey [26].

Fig. 3 FOMBT results of the $\mathrm{P}_{3}$ and $\mathrm{P}_{4}$ Stokes parameters for neon ${ }^{1} \mathrm{P}_{1}$ (denoted as $\mathrm{sP1}$ ) and ${ }^{3} P_{1}$ (denoted as $\mathrm{tP} 1$ ) excitation for $E=20,30,50$, and $80 \mathrm{eV}$ incident electron energies ( $P$ sub 1 is $P_{1}$ and $P$ sub 2 is $P_{2}$ ). The notation for the various energies is given in the lower panel on the left side.

Fig. 4 Differential cross sections for the electron impact excitation of krypton for $\mathrm{E}=50 \mathrm{eV}$ incident electron energy.

Solid line: Present results

Shaded regions: Experimental results of Trajmar et al. [30].

Fig. 5 Spin-orbit coupling effect in neon DCS

Upper panel: $\mathrm{a}^{2}$ and $\mathrm{b}^{2}$ coefficients for rare gases [See Eqs. (1a, b)]

Lower panel: LS-coupled and spin-orbit coupled results for the $\mathrm{Ne} J=1$ excitation DCS-s.

Heavy solid line: Spin-orbit coupled results.

Solid line with empty circles: LS-coupled results.

Shaded region: Experimental results of Register, et al. [28]. 
Fig. 6 Spin-orbit coupling effect in the $P_{3}$ Stokes parameter for neon and argon.

Solid line: Spin-orbit coupled results for ${ }^{3} \mathrm{P}_{1}$ excitation

Dotted line: LS-coupled results for ${ }^{3} \mathrm{P}_{1}$ excitation.

Dashed line: Spin-orbit coupled results for ${ }^{1} \mathrm{P}_{1}$ excitation

Upper panel: FOMBT results for neon

Lower panel: FOMBT results for argon

Fig. 7. Principal quantum number (n) dependence of the $P_{1}, P_{2}$, and $P_{3}$ Stokes parameters for the excitation of the $n s^{\prime}[1 / 2]_{1}^{0}(n=3,4,5$, and 6$)$ states of neon.

Fig. 8 Atomic number $(Z)$ dependence of the DCS for the excitation of the $n s^{\prime}[1 / 2]_{1}^{0}$ states of rare gases.

Fig. 9 Atomic number (Z) dependence of the $\gamma$ (GAMMA) and $\mathrm{L}_{\perp}$ (L-perp) EICP's for the $n s^{\prime}[1 / 2]_{1}^{0}$ excitation of the rare gases.

Fig. 10 Atomic number $(Z)$ dependence of the $P_{3}$ Stokes parameter for the $n s^{\prime}[1 / 2]_{1}^{0}$ excitation of the rare gases.

Fig. 11 Atomic number (Z) dependence of the $\rho_{o o}$ (rho00) EICP for the ns' $[1 / 2]_{1}^{0}$ excitation of the rare gases.

Fig. 12 Atomic number $(Z)$ dependence of the $P_{1}$ and $P_{2}$ Stokes parameters for the $n s^{\prime}[1 / 2]_{1}^{0}$ excitation of the rare gases.

Solid line: DWA results of Bartschat and Madison [31]

Dashed line: FOMBT results from:

For He: Csanak and Cartwright $[33,34]$

For Ne: presnet results

For Ar: da Paixão, et al. [12]

For Kr: Solid circles: Zheng and Becker [32]

Solid squares: Danjo, et al. [36] 


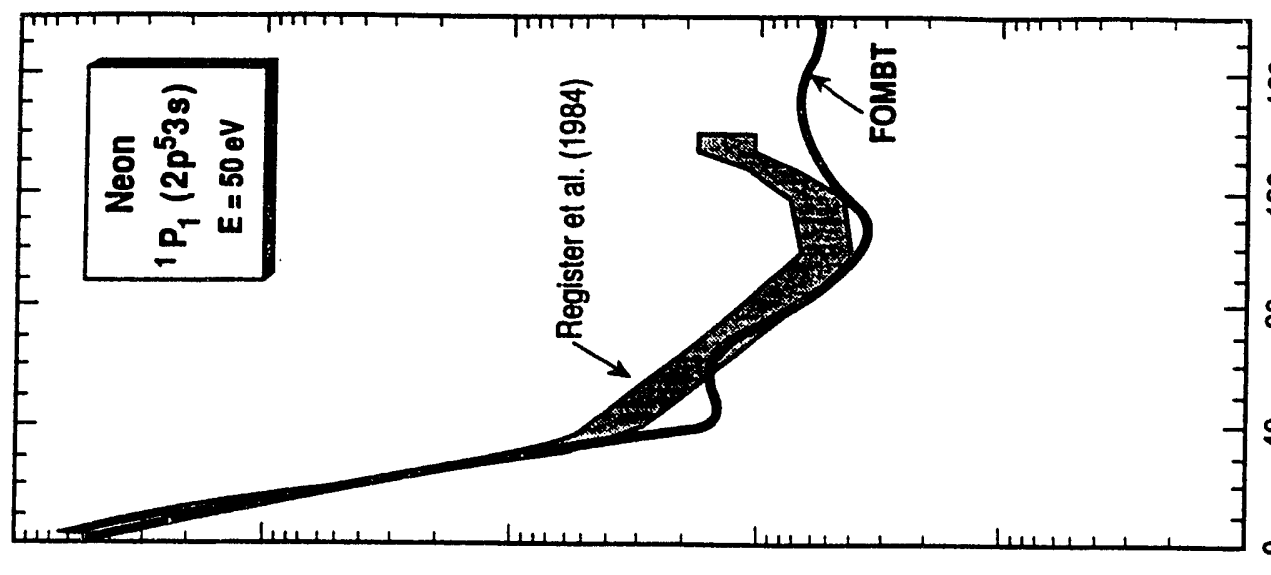

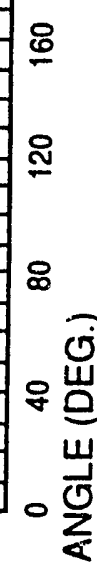
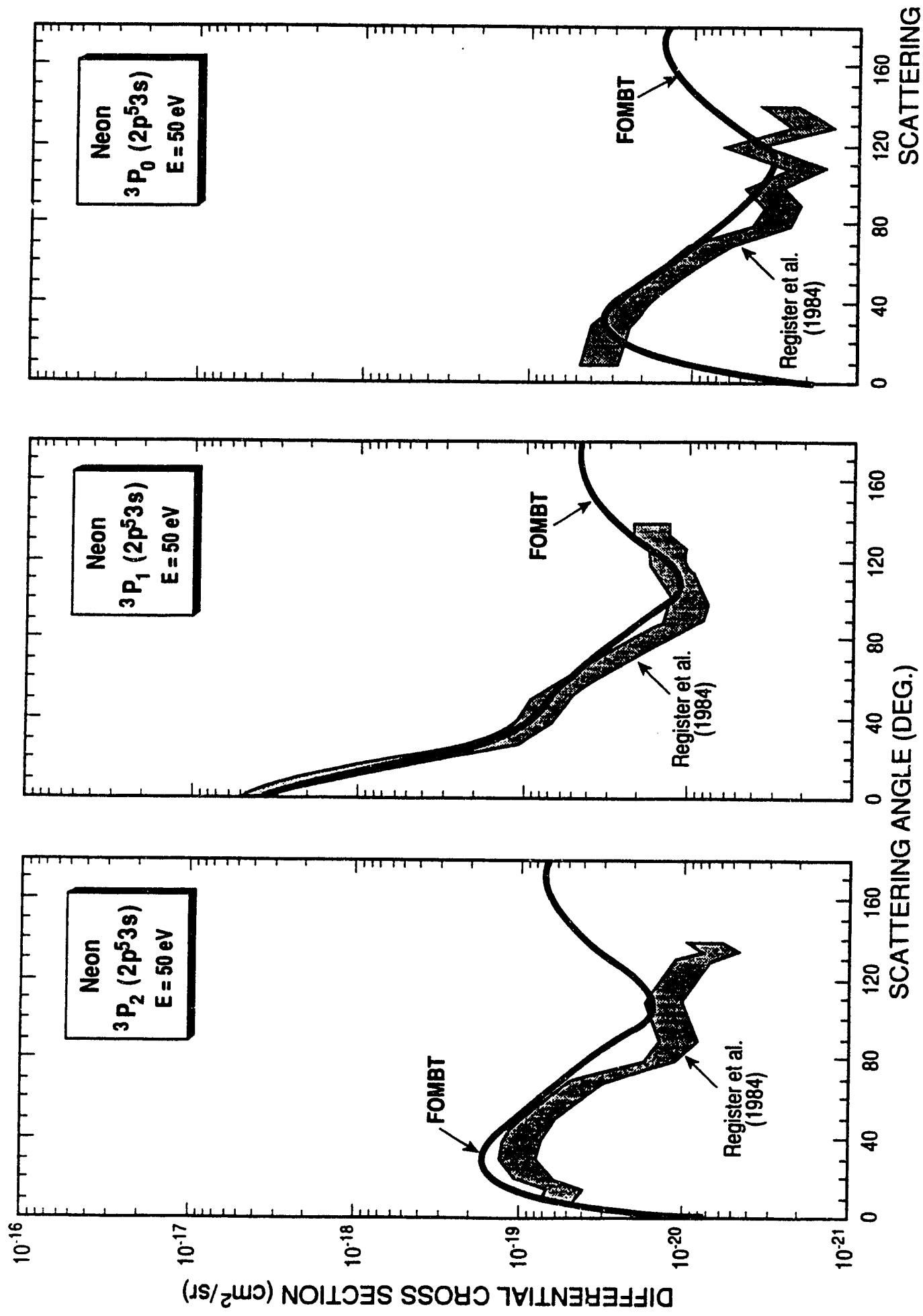

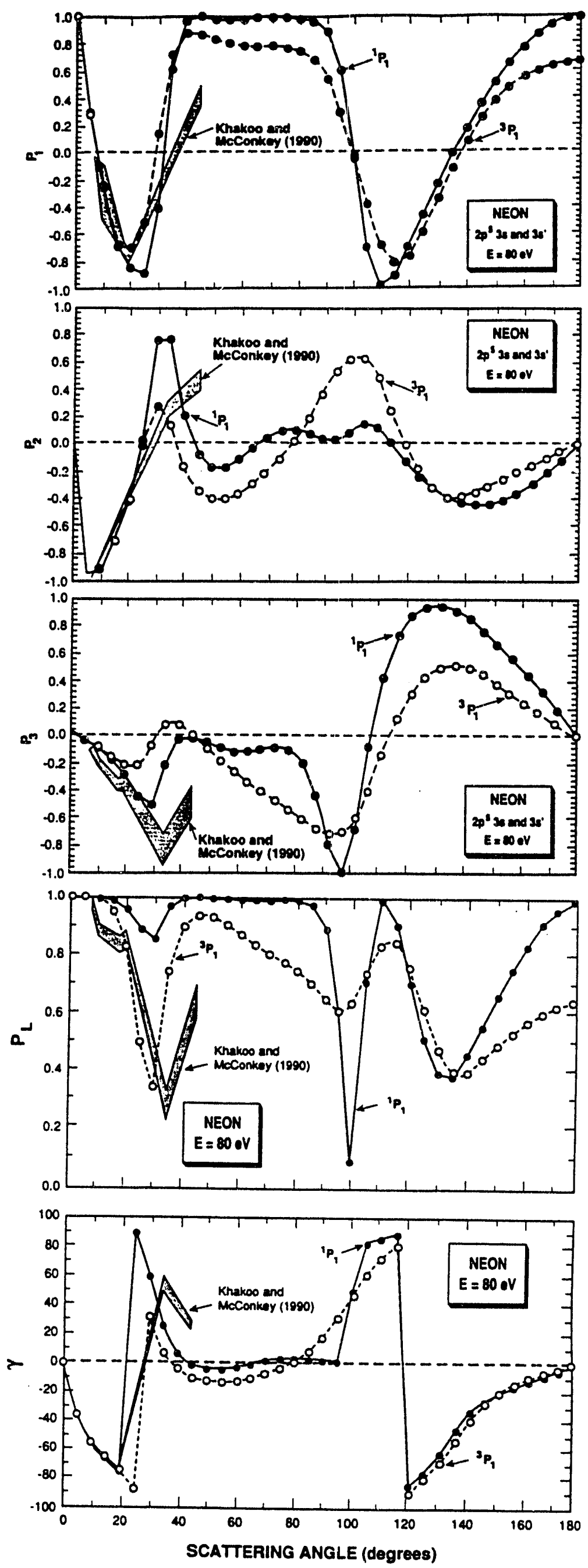


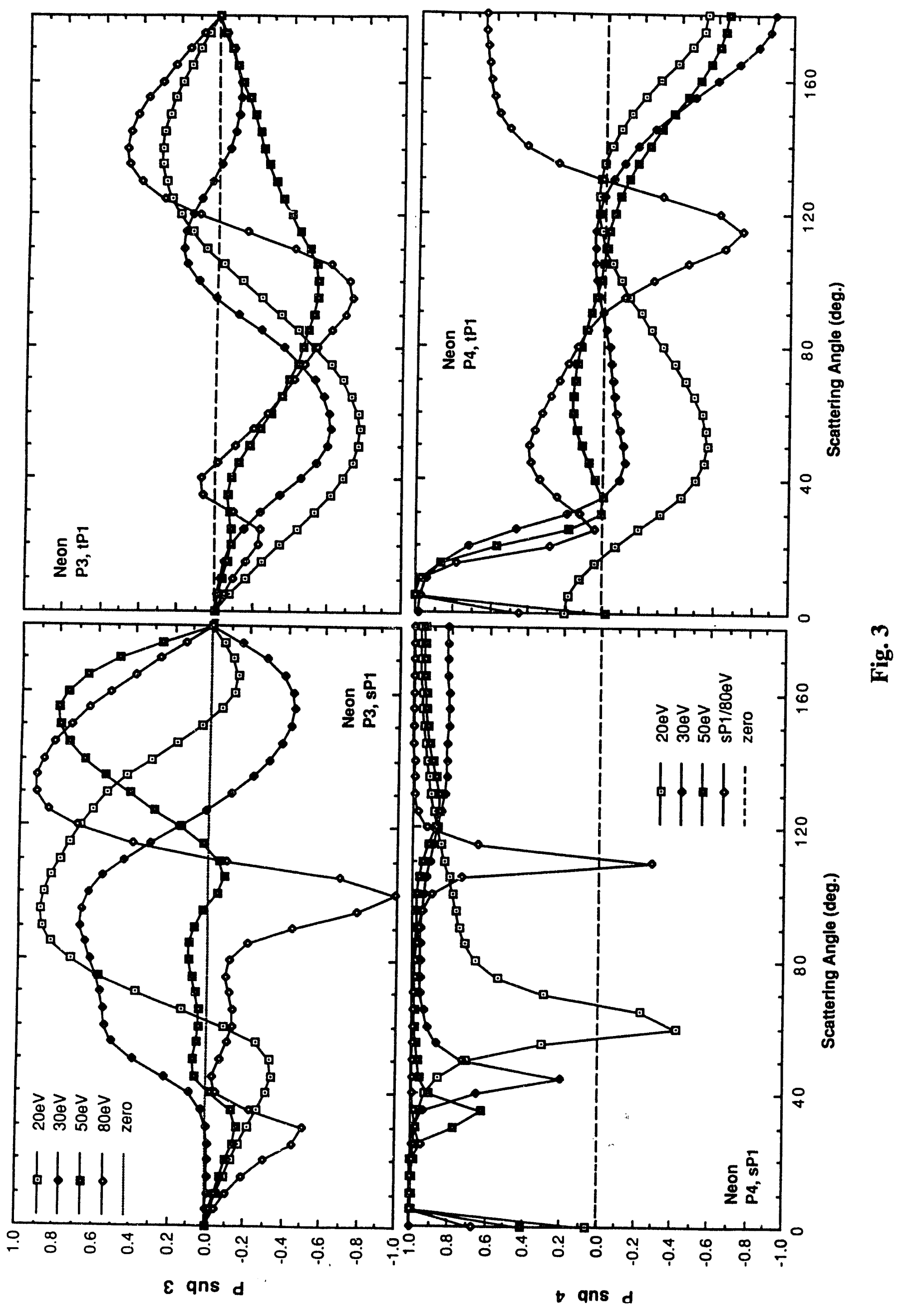




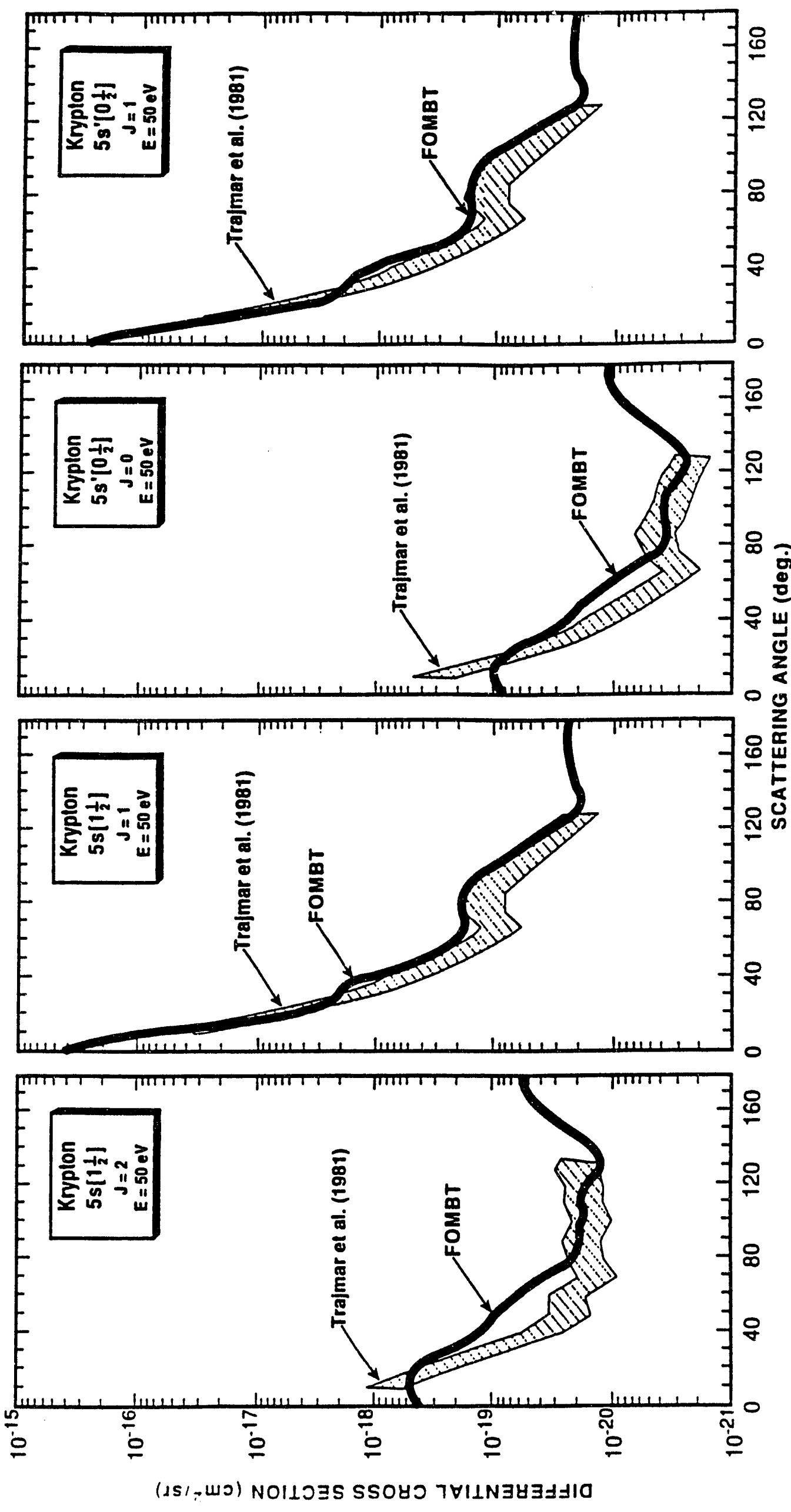



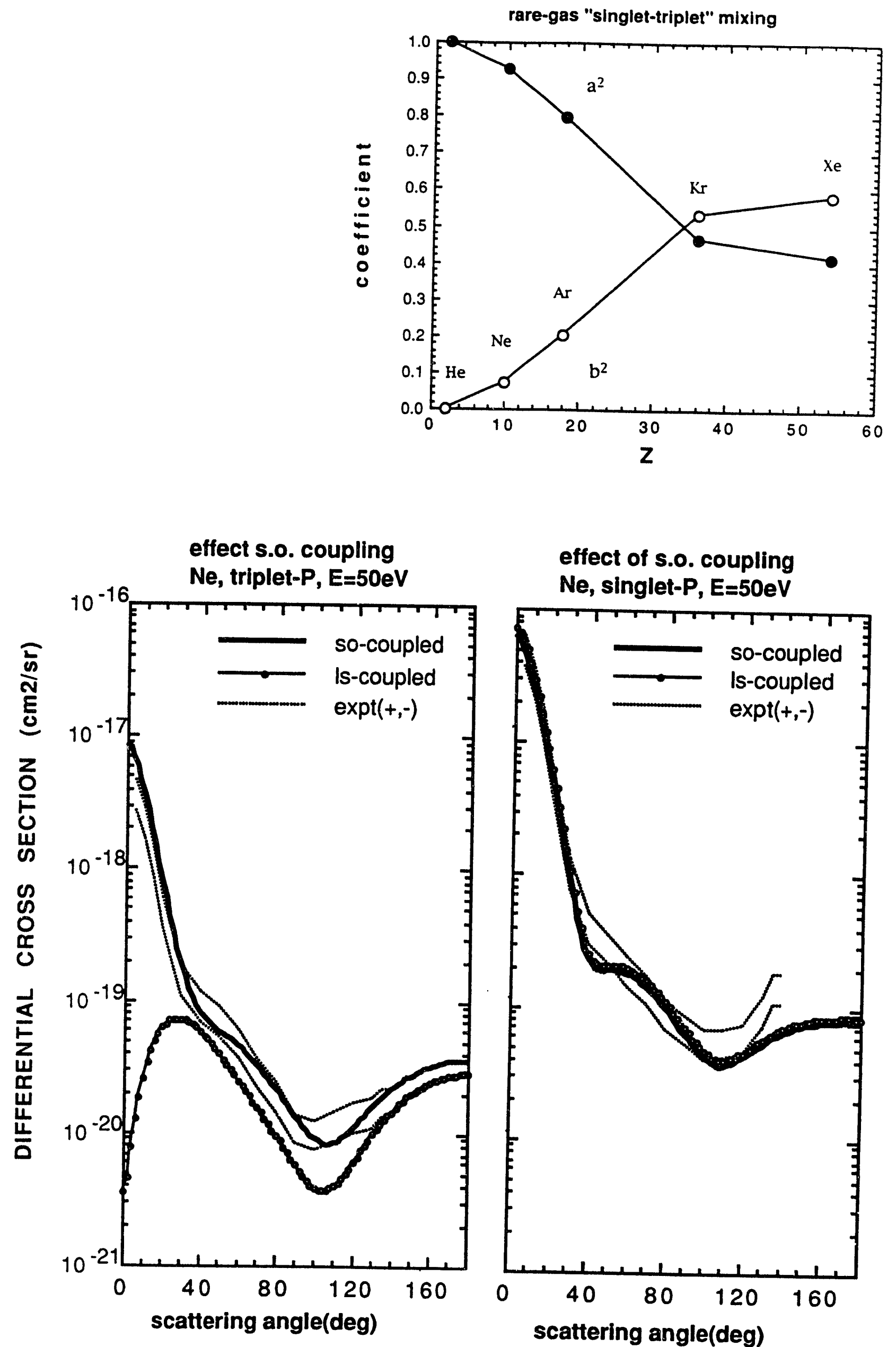

effect of s.o. coupling $\mathrm{Ne}$, singlet-P, $E=50 \mathrm{eV}$

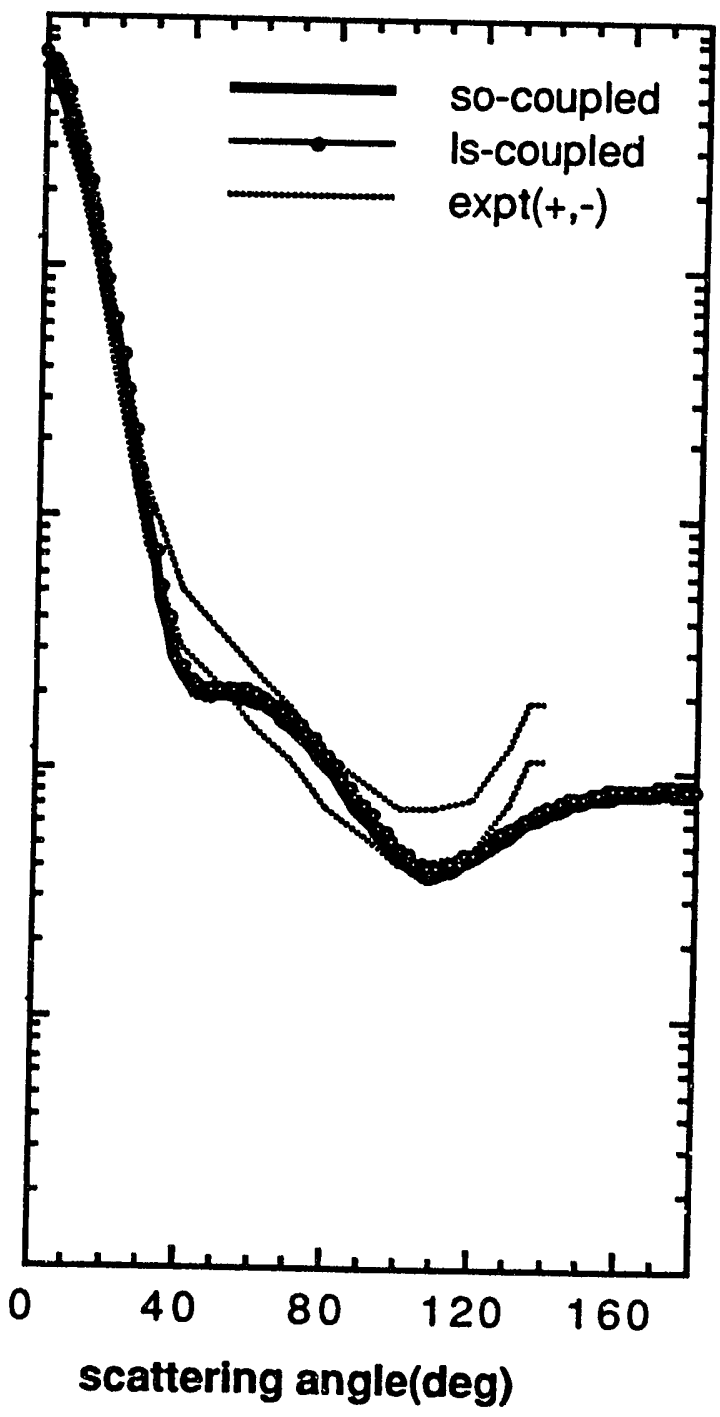

Fig. 5 
spin-orbit coupling in Ne, triplet-P, $E=50 \mathrm{eV}$

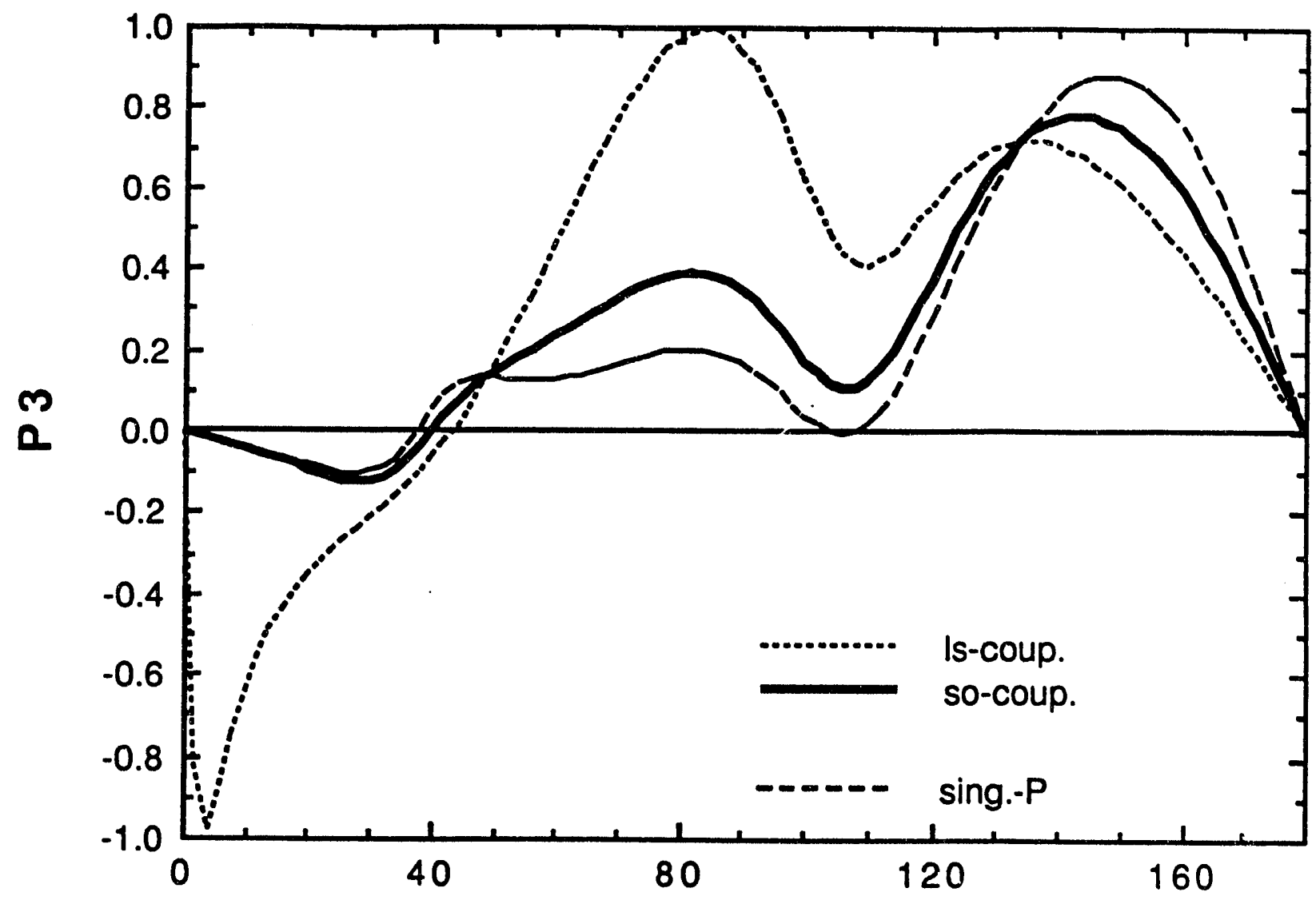

spin-orbit coupling in Ar, triplet-P, $E=50 \mathrm{eV}$

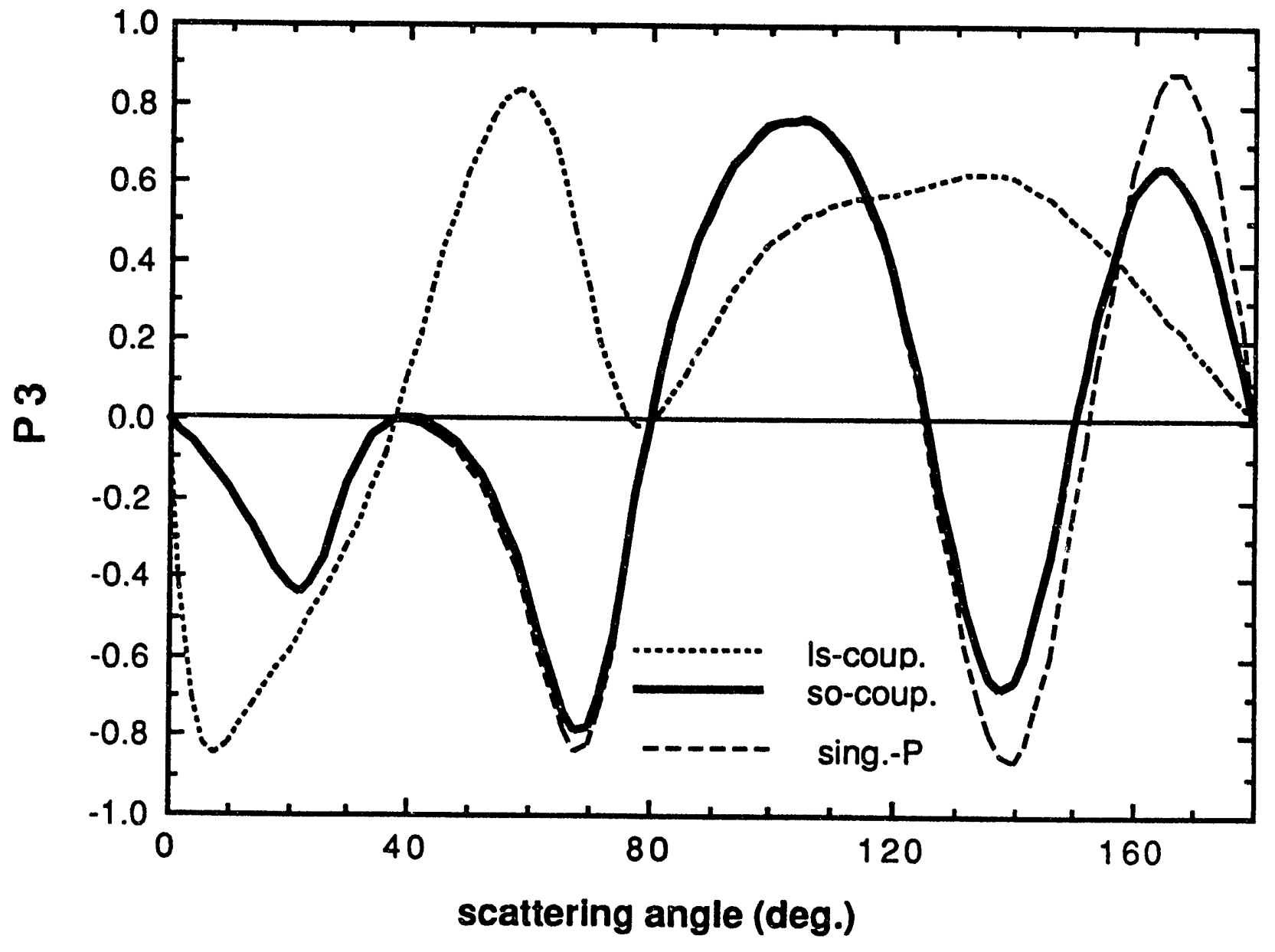

- Fig. 6 


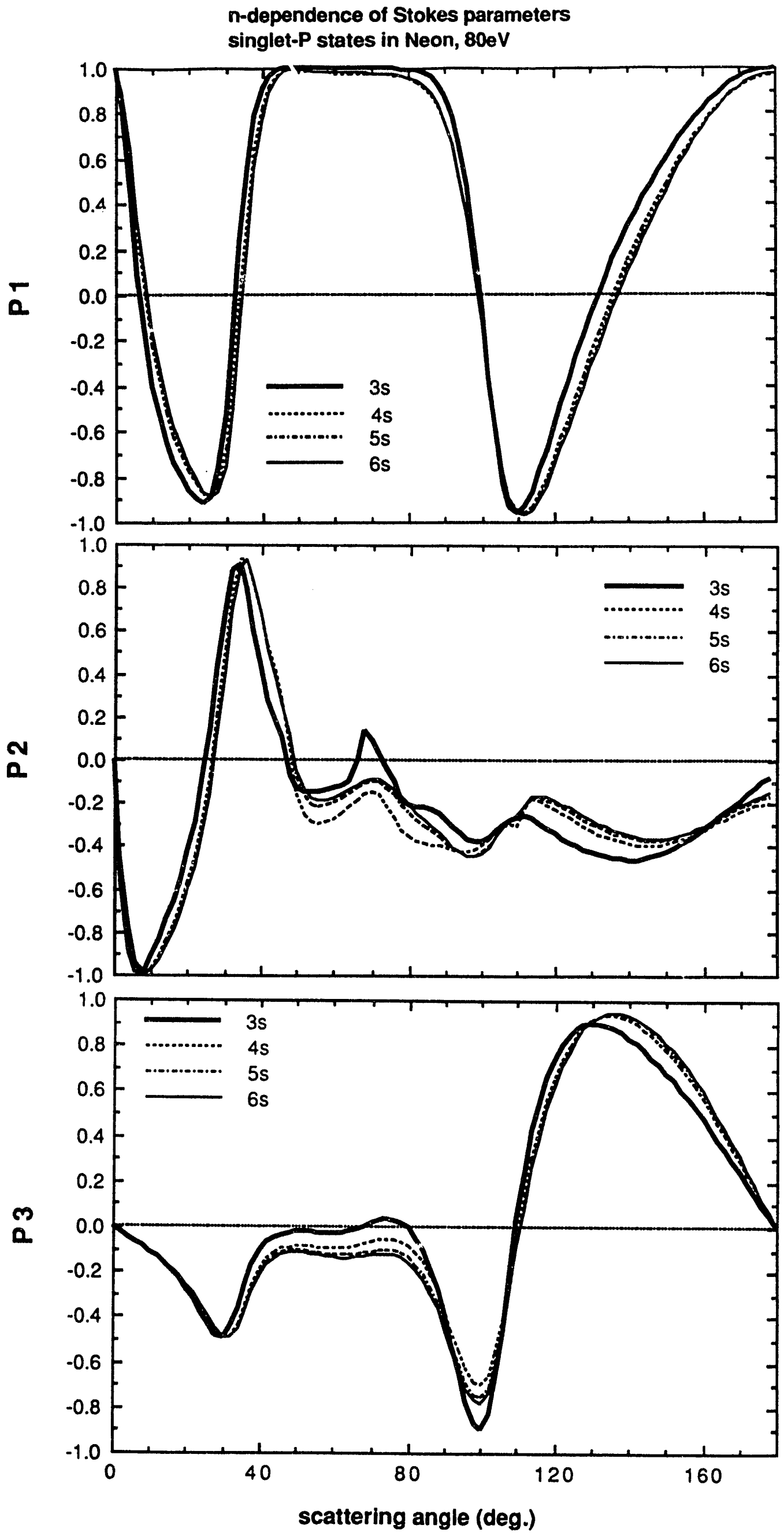

Fig. 7 


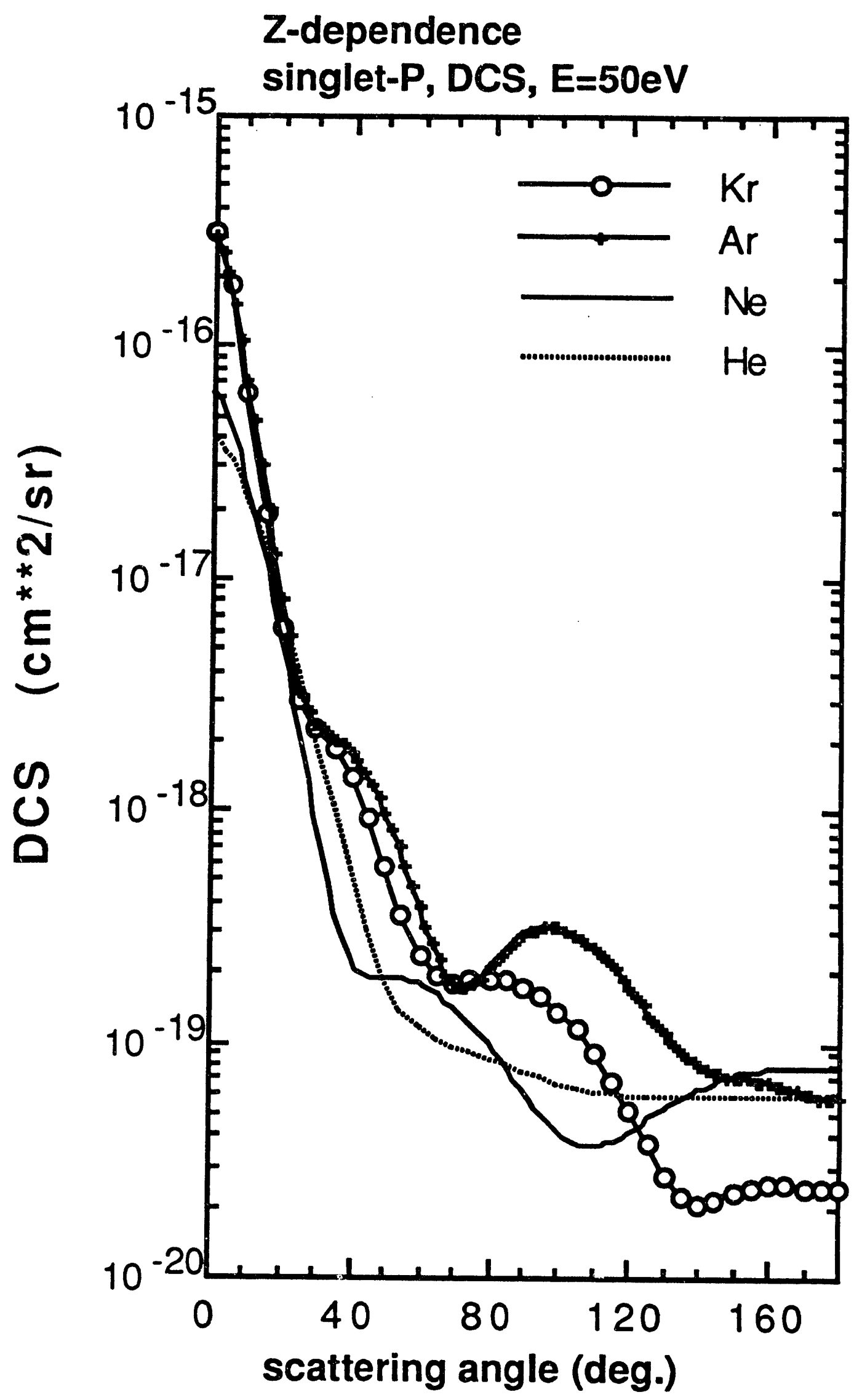

Fig. 8 

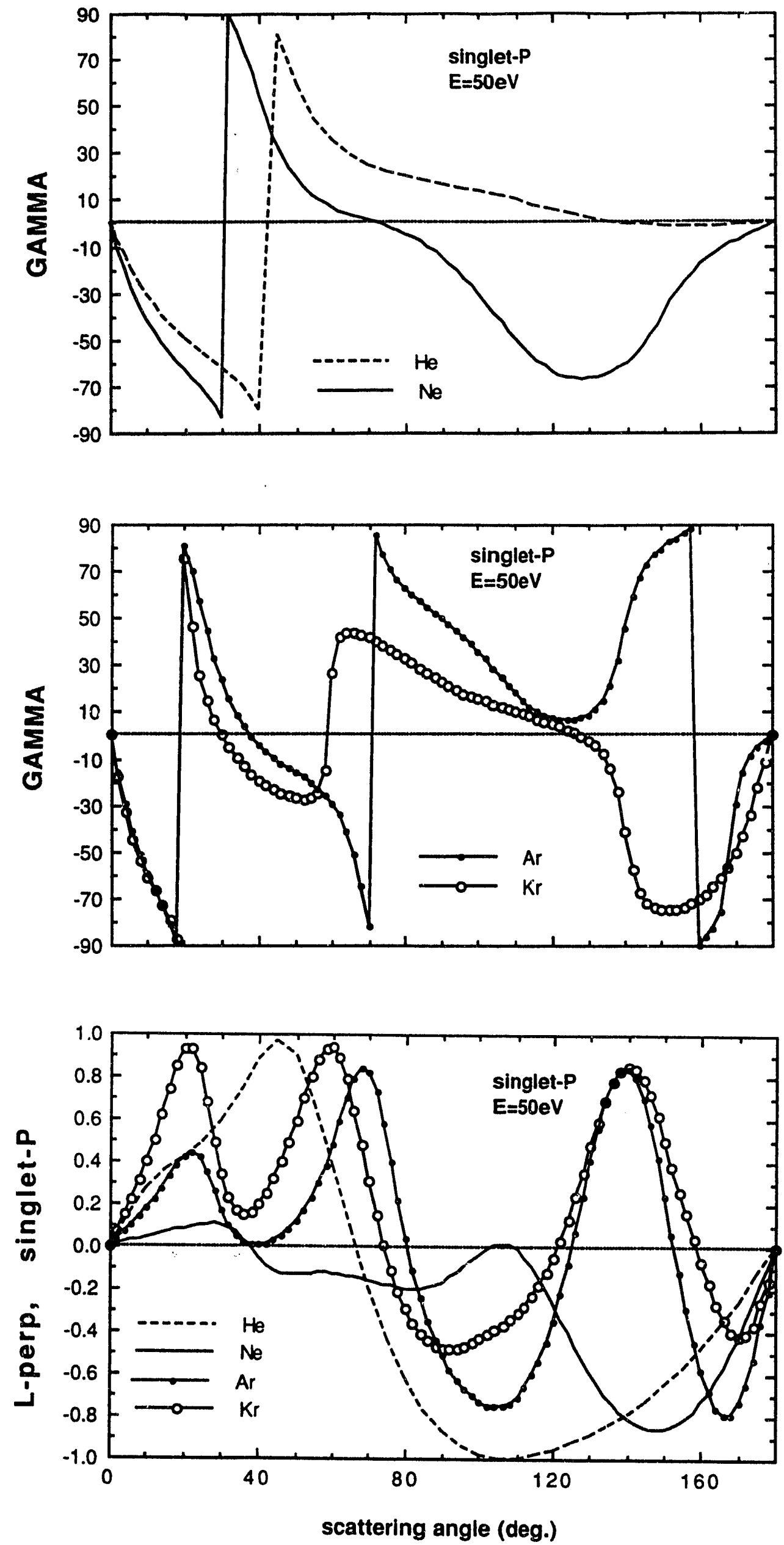

Fig. 9 


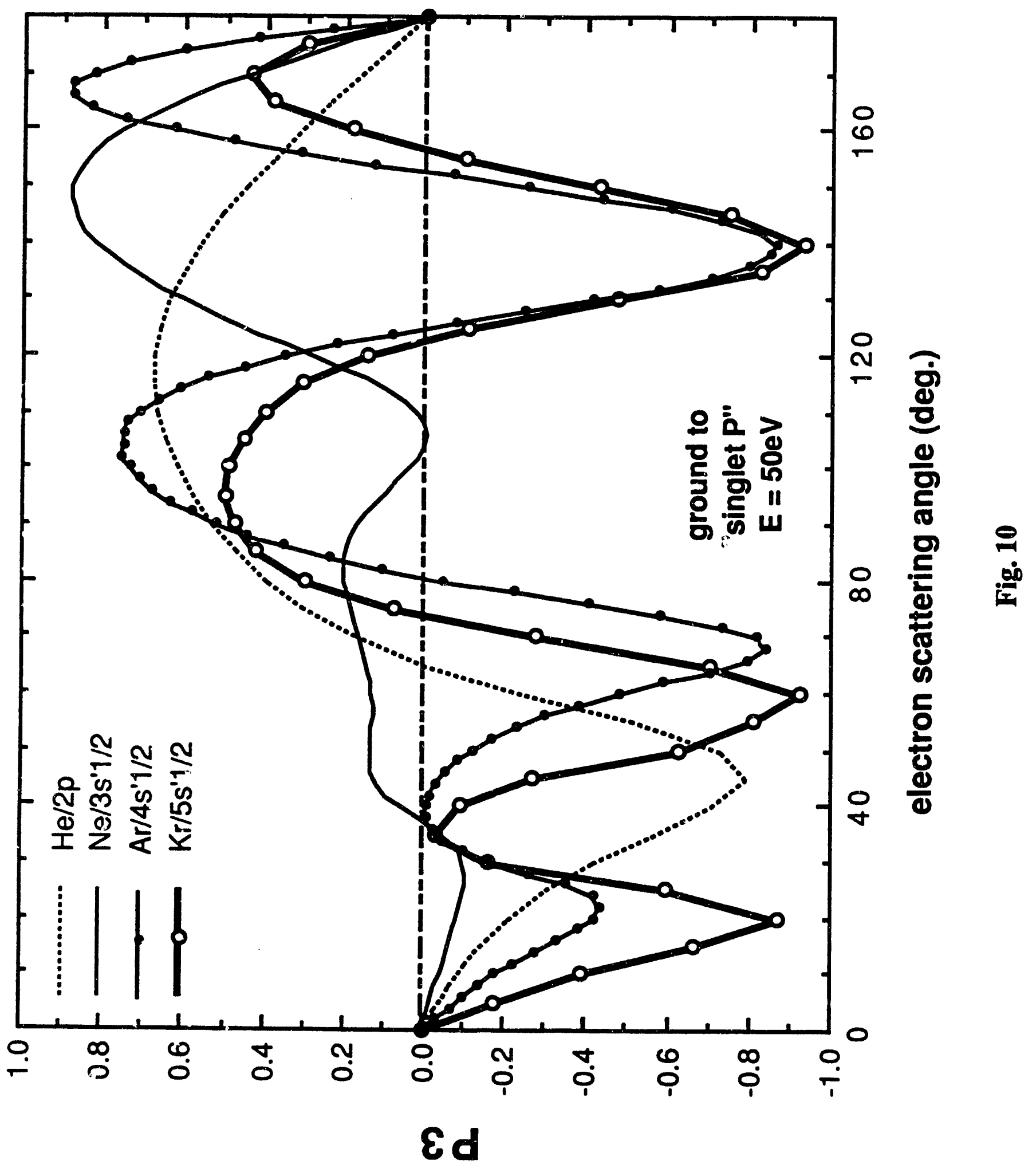




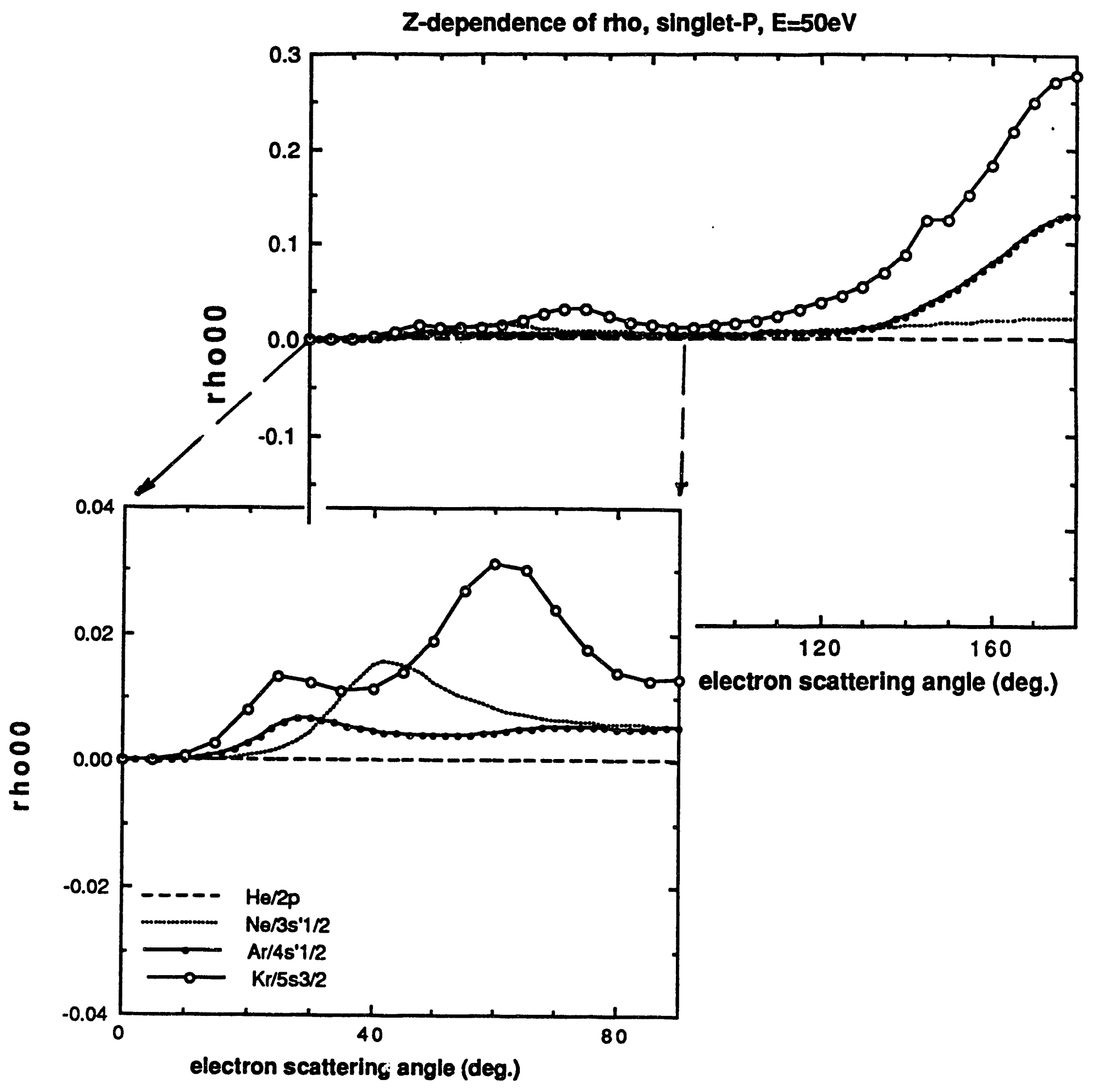

Fig. 11 
L-aepencence at $50 \mathrm{eV}$

$P_{1}$ parameter

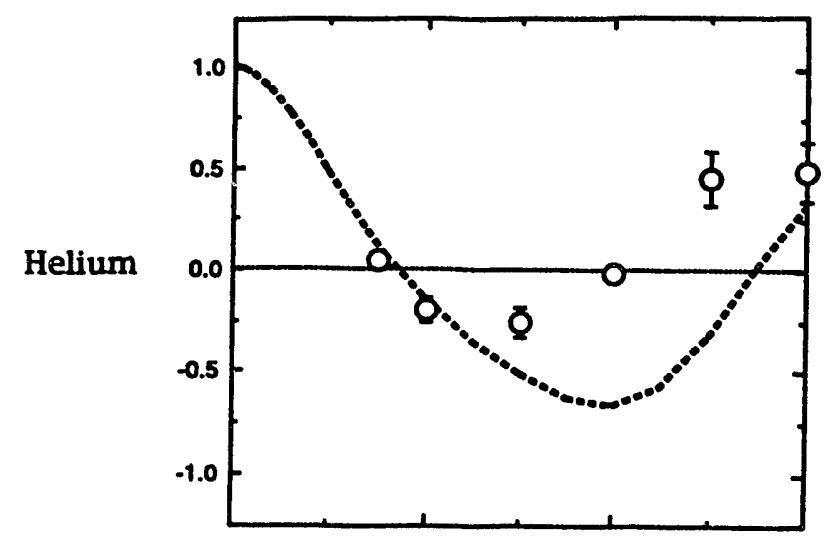

$\mathbf{P}_{\mathbf{2}}$ parameter
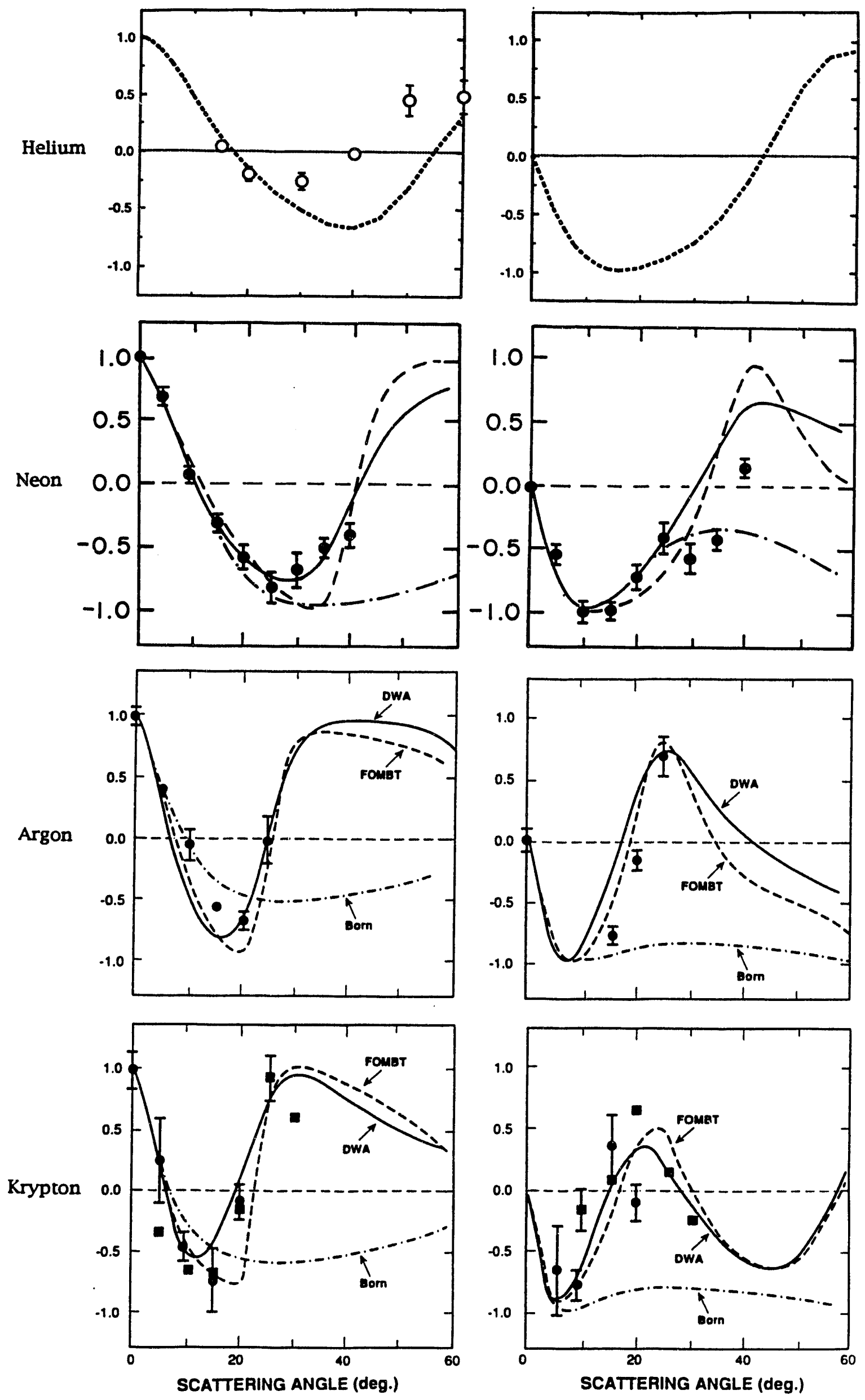

Fig. 12 

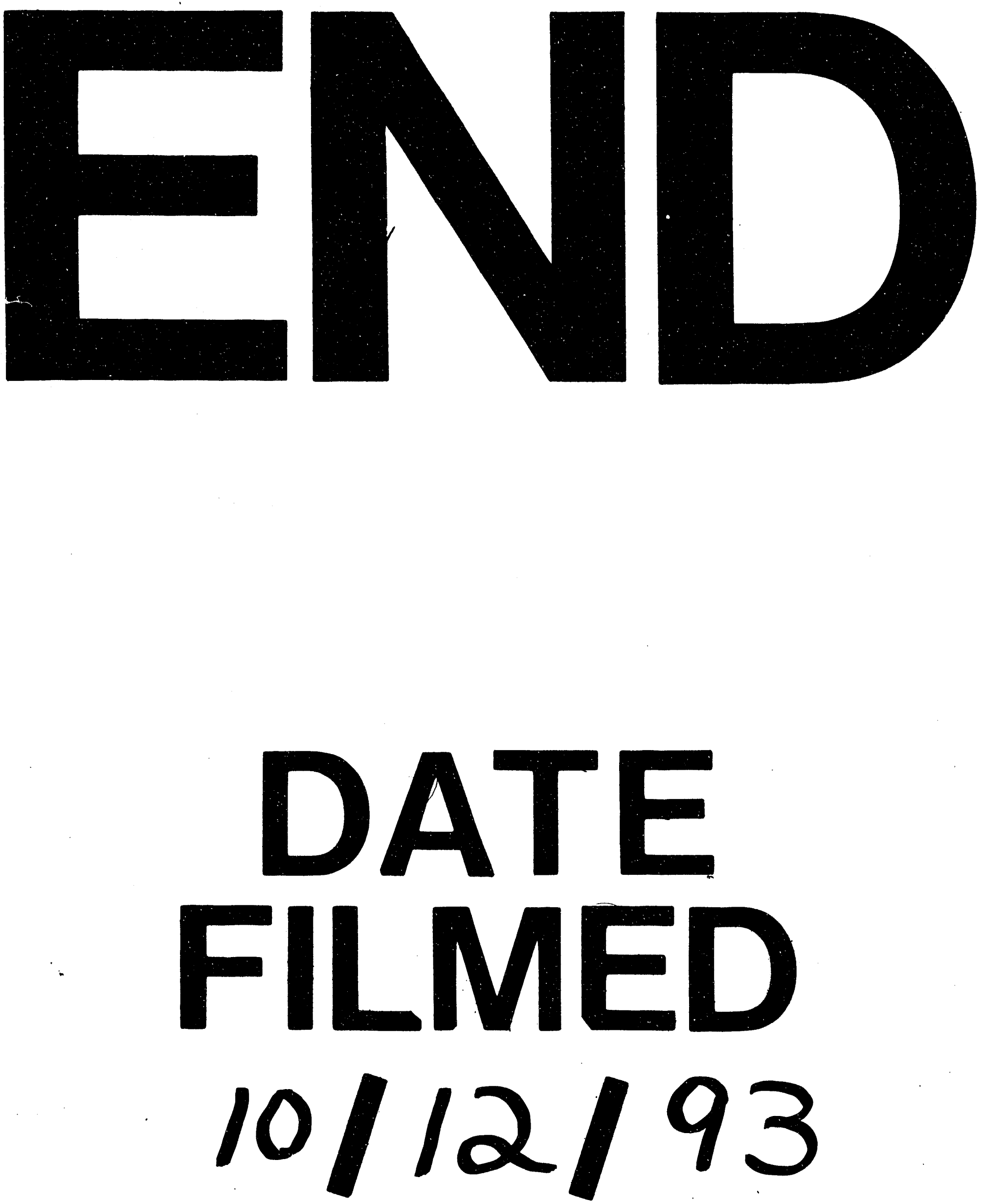

1 
